\title{
A Simple Novel Expedited Spike Culture-derived Variation Creation Strategy in Wheat
}

\author{
S. GANESHAN ${ }^{1}$ and R.N. CHIBBAR ${ }^{2 *}$ \\ ${ }^{1}$ Biotechnology Laboratories, Saskatchewan Research Council, 125-15 Innovation Boulevard, \\ Saskatoon, SK, Canada \\ ${ }^{2}$ Department of Plant Sciences, University of Saskatchewan, College of Agriculture \& Bioresources, \\ 51 Campus Drive, Saskatoon, SK, Canada
}

(Received 7 November 2016; Accepted 5 May 2017)

\begin{abstract}
A wheat (Triticum aestivum L.) immature spike culture system was used to expeditiously generate mutations for use in wheat improvement programs. Wheat immature spikes in culture were treated with three concentrations of ethylmethane sulphonate (EMS) to generate a spike culture derived variant (SCDV) population. EMS in a concentration dependent manner affected seed development in wheat immature spike cultures. Based on the number of seeds produced, inclusion of EMS $(25 \mathrm{mM})$ for three hours in immature spike culture medium generated variants in wheat cv. AC Nanda. The wheat AC Nanda SCDV population showed variation in several phenotypic characters. Flag leaf (length, angle and sheath length), length of first and second internode, spike length, number of spikes, number of seeds per spike and seed weight, showed variation below and above the non-treated controls. A molecular screening technique combining simple sequence repeat (SSR) oligonucleotide primers with high resolution melt (HRM) PCR with EvaGreen was used to identify the variants. Screening for starch branching enzyme IIb (SbeIIb) revealed 75 lines with point mutations. Combining SSR and SbeIIb, a total of $100 \mathrm{Kbp}$ portion of wheat DNA was screened. The estimated mutation frequency in SbeIIb was one per $20.8 \mathrm{Kbp}$. The spike culture system utilizes very small amounts of EMS for a brief period, thus needs minimal handling of EMS and saves one generation of plant growth in a greenhouse. The morphological variants observed are similar to those reported for seed-derived variants using EMS.
\end{abstract}

Keywords: ethyl methane sulphonate, mutation, wheat, high resolution melt curve

\section{Introduction}

The success of crop improvement through breeding is dependent on new sources of variation in desirable traits for incorporation into new cultivars. However, dwindling variability within existing germplasm has become a major impediment to expedite breeding efforts to enhance agronomic performance and improve grain quality (Chibbar et al. 2007; Jansson et al. 2007). Chemical mutagenesis has been used to generate variability to complement breeding programs and also to help in basic research in functional genomics research in a forward genetic approach. Mutation breeding practiced since the 1930s has 
made a tremendous impact on crop improvement. The mutant varieties database of the IAEA/FAO lists over 3000 varieties of plants released as a result of mutagenesis (http:// mvgs.iaea.org/). Chemical mutagenesis has resulted in 349 varieties including 38 varieties each of wheat and barley, that were either directly released as varieties or the mutated germplasm was incorporated into breeding programs for subsequent release as varieties (http://mvgs.iaea.org/). However, the mutation generation process based on seed mutagenesis is resource-demanding, time-consuming and generates numerous wasteful mutants. For example, depending on the concentration of a mutagen such as ethylmethane sulphonate (EMS), many lethal mutants may be generated, precluding identification of useful mutant phenotypes. With currently available high-throughput genomics resources, it is now possible to rapidly screen for desired mutations for rapid incorporation into breeding programs. The targeting induced local lesions in genomes (TILLING) (McCallum et al. 2000a; Till et al. 2003) has been used to identify useful variants in wheat. In addition, the possibility of high-throughput screening for mutation by high resolution melt (HRM) analysis (Ririe et al. 1997) further expedites identification of mutations for traits where variability has been limiting. More recently exon-capture methods have been used for identification of mutations (Henry et al. 2014; King et al. 2015), adding to the repertoire for mutation screening.

Although high throughput methods to screen mutant lines are available, the challenge still remains the generation of manageable useful mutants in wheat. We recently developed a versatile cereal spike culture system for studying grain development (Ganeshan et al. 2010) and cadmium accumulation in grains (Ganeshan et al. 2011). In this report spike culture has been used to expeditiously develop wheat variant populations. The conventional mutagenesis approach uses seeds as starting material and the plants $\left(\mathrm{M}_{1}\right)$ are grown, seeds are collected and grown from individual heads for subsequent molecular analysis of $\mathrm{M}_{2}$ plants, with the M3 seeds catalogued for future use. Compared to the conventional seed mutagenesis, the inclusion of mutagenic agent like EMS in the spike culture system prior to occurrence of anthesis (Fig. 1a) increases the likelihood of the germ-line cells being mutated and leading to more mutation events in the developing seeds. In wheat TILLING populations have been developed (Slade et al. 2005; Dong et al. 2009; Uauy et al. 2009), and used to identify variations in waxy gene (Slade et al. 2005), starch synthase IIa (Hogg et al. 2013), waxy and starch synthase II (Sestili et al. 2010), rust and powdery mildew resistance (Campbell et al. 2012), and other morphological traits (Dhaliwal et al. 2015). Due to the seed mutagenesis being laborious and requiring large volumes of mutagen solution, a simple and rapid alternative would be valuable to expedite crop improvement. For example, to treat 300-350 wheat seeds, $100 \mathrm{~mL}$ of EMS was used (Dong et al. 2009; Chen et al. 2012). Furthermore, due to concerns such as disposal and exposure with handling of chemical mutagens, the immature spike culture system reduces such risks due to minimal volumes of mutagenic agent required per spike. 


\section{Materials and Methods}

\section{Plant growth, mutagen treatment and spike culture establishment}

The soft white spring wheat (Triticum aestivum L.) cv. AC Nanda (Sadasivaiah et al. 2000) was used for mutation generation in immature spikes. Plants were grown in a growth chamber in pots containing Redi-earth (W.R. Grace \& Co. of Canada, Ontario, Canada) at $23{ }^{\circ} \mathrm{C} / 16 \mathrm{~h}$ light $\left(350 \mu \mathrm{mol} \mathrm{m} \mathrm{m}^{-2} \mathrm{~s}^{-2} \mathrm{PPFD}\right)$ and $19{ }^{\circ} \mathrm{C} / 8 \mathrm{~h}$ dark. Plants were fertilized every three weeks with slow release fertilizer, Nutricote ${ }^{\mathrm{TM}}-14-14-14: \mathrm{N}-\mathrm{P}-\mathrm{K}$ (Plant Products Co. Ltd., Brampton, ON, Canada).

For spike culture establishment, immature spikes were cut above the soil surface when awns started to emerge from the leaf sheath. All the leaves and flag leaves were removed (Fig. 1a). The sheaths were left intact and undisturbed. Excised spikes were placed in tubes containing $2 \mathrm{~mL}$ of culture medium supplemented with ethyl methanesulphonate (EMS) (Sigma-Aldrich, ON, Canada). Three EMS concentrations (10, 25 and $50 \mathrm{mM})$ were used in the culture medium to treat three immature spikes for each concentration for three hours. The spikes were then transferred to spike culture medium without EMS. The spikes were transferred to fresh culture medium every four days, after excising a few millimeters to a centimeter from their bases. Thus, during the course of an experiment, spike length was reduced. Furthermore, if browning of the peduncular tissues occurred in the medium, these were promptly excised. The culture medium ( $\mathrm{pH}$ 6.2) consisted of $50 \mathrm{~g} \mathrm{~L}^{-1}$ sucrose and $0.4 \mathrm{~g} \mathrm{~L}^{-1} \mathrm{~L}$-glutamine, buffered with $0.5 \mathrm{~g} \mathrm{~L}^{-1}$ morpholino ethane sulfonic acid (as per Ganeshan et al. 2007). The spikes were allowed to develop to maturity (Ganeshan et al. 2010). Seeds $\left(M_{0}\right)$ were collected and planted ( $M_{1}$ plants). Six thousand $M_{1}$ seeds were obtained and constituted the SCDV population. Sixty seeds were randomly selected from the SCDV lot and planted, 55 of which produced $\mathrm{M}_{2}$ plants.

\section{DNA isolation and high resolution melt (HRM) curve analysis}

DNA was isolated from leaf tissues using the PureLink ${ }^{\circledR}$ Genomic DNA extraction kit (Life Technologies, Inc., Burlington, ON) as per the manufacturer's instructions. For HRM analysis, $100 \mathrm{ng}$ of DNA was used with $2 \mathrm{X}$ MeltDoctor ${ }^{\mathrm{TM}}$ HRM Master Mix (Applied Biosystems, Inc., Burlington, ON, Canada) containing $300 \mathrm{nM}$ of the respective primers in a total volume of $20 \mu \mathrm{L}$. The amplification was performed using a 7500 Fast real-time PCR system with HRM capability (Applied Biosystems, Inc., Burlington, ON, Canada). Simple sequence repeats (SSR) primers as well as primers specific to the SbeI$I b$ gene were used to screen for variants within the amplified regions (Table S1*). SSR and SbeIIb specific amplification conditions were as follows: $95^{\circ} \mathrm{C} / 1 \mathrm{~min} ;\left(95^{\circ} \mathrm{C} / 10 \mathrm{~s}\right.$, $\left.55^{\circ} \mathrm{C} / 1 \mathrm{~min}\right) \times 40$, followed by high resolution melt curve from $95{ }^{\circ} \mathrm{C}$ to $60{ }^{\circ} \mathrm{C}$, at $1 \%$ ramp rate.

*Further details about the Electronic Supplementary Material (ESM) can be found at the end of the article. 

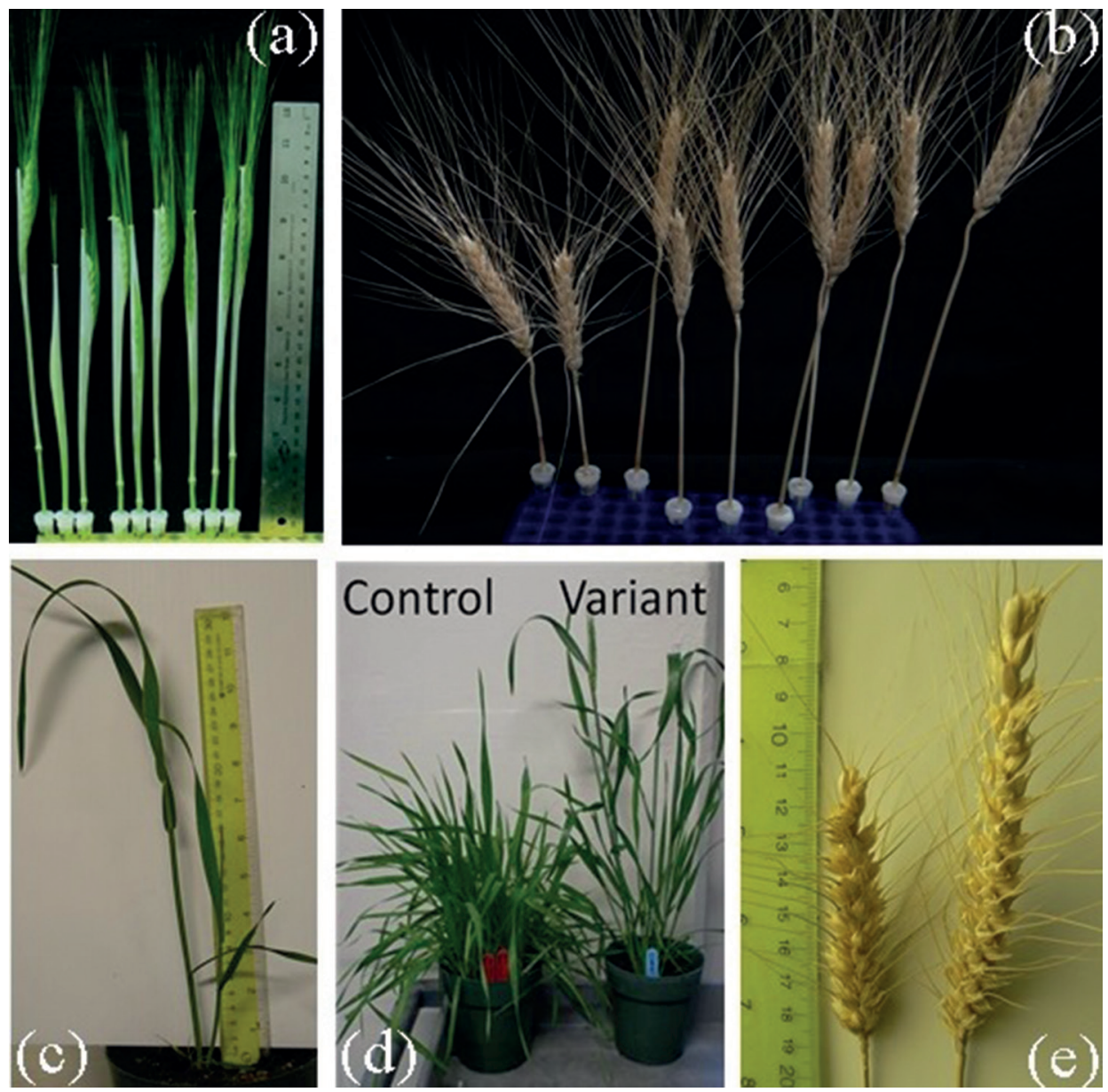

Figure 1. EMS treatment of wheat and growth of progeny derived from grains of EMS treated spikes. (a) Immature spikes prior to anthesis in culture medium containing different concentrations of EMS; (b) EMS treated spikes cultured to maturity; (c) $\mathrm{M}_{2}$ plant showing only two tillers; (d) Variant line heading after 45 days compared to wild-type control plant; (e) Wild-type spike (left) compared to extended head (right) from a variant line

\section{Results}

\section{Variant population establishment}

The in vitro spike culture system utilized $2 \mathrm{~mL}$ of culture medium (Ganeshan et al. 2010), and its supplementation with 10, 25 and $50 \mathrm{mM}$ EMS for $3 \mathrm{~h}$ prior to anthesis (Fig. 1a) was used to generate mutants. The selected concentrations of EMS were based on concentrations used for wheat seed mutagenesis. The duration of treatment was previously opti- 
mized using fluorescent dye uptake studies (data not shown), which showed that dye reached the top-most wheat spikelets in $3 \mathrm{~h}$. Longer exposures $(>5 \mathrm{~h})$ to the EMS using the spike culture led to empty caryopses, indicating toxicity. After $3 \mathrm{~h}$ of EMS treatment the spikes were transferred to spike culture medium without EMS and allowed to develop to maturity (Ganeshan et al. 2010). Each spike, depending on EMS concentration used, produced 10 to 20 viable seeds compared to 20 to 30 seeds in a non-treated control spike in culture. The EMS concentration affected seed production in a dose dependent manner. The number of $\mathrm{M}_{1}$ seeds obtained with 10, 25 and $50 \mathrm{mM}$ EMS were 3851, 2492 and 324, respectively. This showed that the higher concentration of EMS, as would be expected, is lethal, with reduced seed set. The intermediate concentration of $25 \mathrm{mM}$ EMS was found to be optimal.

\section{Morphological traits}

$\mathrm{M}_{1}$ and $\mathrm{M}_{2}$ plants have exhibited a range of phenotypes from dwarf to late heading to extended heads (Fig. 1b-e). To determine if the EMS treatment adversely affected certain morphological characteristics, flag leaf length, flag leaf sheath length, flag leaf angle, length of first internode, length of second internode, number of seeds per spike, weight of seeds per spike and number of spikes on $\mathrm{M}_{2}$ plants were measured.

Extreme variations were observed for some of the traits. The flag leaf length, for example, ranged from 7.2 to $25.3 \mathrm{~cm}$, with the control plant intermediate at $17.4 \mathrm{~cm}$ (Fig. S1). Variation in the flag leaf angle was also apparent ranging from 12 to $61.5^{\circ}$, with the wild-type flag leaf angle at $26.3^{\circ}$ (Fig. S1). Interestingly, most of the variants had greater flag leaf angle than the wild-type. On the other hand, flag leaf sheath length showed less variability and only three variants were comparable to the wild-type (Fig. S1).

Variation in parameters such as the spike (ear) length, lengths of first internode (from bottom of spike) and second internode was also observed (Fig. S2). Only four variants showed second internode length (11.4-12.4) shorter than the wild-type $(13 \mathrm{~cm})$, the remaining were longer, with the longest at $20 \mathrm{~cm}$ (Fig. S2). However, with the first internode, most of the variants were shorter $(7.9-19.1 \mathrm{~cm})$ than the wild-type $(19.2 \mathrm{~cm})$, with the maximum length being $26.5 \mathrm{~cm}$. Distribution of the spike length was similar to the wild-type $(9.7 \mathrm{~cm})$, with the shortest at $7.2 \mathrm{~cm}$ and the longest at $15.7 \mathrm{~cm}$ (Fig. S2).

Two other traits, number of seeds per spike and weight of seeds per spike, were also recorded (Fig. S3). The number of seeds per spike was highly variable, with some variants showing very low seed set. While the wild-type had an average of 42 seeds per spike, several variants had higher seed set, with a maximum at 50 seeds (Fig. S3). The weight of seeds per spike for the wild-type was intermediate with respect to the variants at $1.2 \mathrm{~g}$. Due to the low seed set in some of the variants, seed weight in the low range was rather skewed. However, in the high range the maximum seed weight was at $2 \mathrm{~g}$ (Fig. S3). 


\section{HRM analysis of variants}

Fifty-five wheat variants were assessed using the 7500 Fast real-time PCR system with HRM capability (Applied Biosystems, Inc.) for mutations in coding regions of SbeIIb. We initially targeted the starch branching enzyme IIb (SbeIIb) gene, since the genomic DNA sequence of wheat gene is available and primers could be designed to span only the exon regions. Furthermore, to-date there are no known naturally occurring mutations for the SbeIIb gene in wheat. By RNAi transgenic silencing strategies, it has been shown that the down-regulation of both SbeIIa and SbeIIb led increased resistant starch (i.e., 70\% amylose) in wheat (Regina et al. 2006). After screening three exon regions (exons 3, 9 and 10), we identified several mutations.

To establish a mutation frequency, we analyzed genomic DNA from the selected mutant lines with SSR markers using HRM (Fig. S4a) allowing detection of single nucleotide polymorphisms (SNP). Screening of SSR primers indicated variations in the mutant lines (Fig. S4a). Similarly, mutations in the gene coding for the wheat Sbellb was also screened by HRM and $2 \times$ EvaGreen master mix (Fig. S4b). The variant lines assessed using HRM for mutations in SbeIIb coding regions were DNA sequenced to verify mutation occurrence (Fig. S4c and d). After screening three exon regions (exons 3, 9 and 10), we identified 75 point mutations. Upon sequencing the amplified fragments from such lines, two fragments showed mutation events in the exons. Combining the SSR and SbeI$I b$ screening and DNA sequencing of a total of $99,660 \mathrm{bp}$, the mutation frequency in the screened $\mathrm{SbeIIb}$ regions was estimated at one mutation per $20.8 \mathrm{Kbp}$.

\section{Discussion}

The new strategy of using immature wheat spike cultures to induce EMS mutations is a simple, rapid and safer alternative to generate mutants from seeds. In vitro spike cultures treated with EMS $(25 \mathrm{mM})$ were able to generate adequate number of viable seeds. The EMS concentration $(25 \mathrm{mM})$ concurs with the recently reported EMS seed mutagenesis in wheat (Dhaliwal et al. 2015). The population derived from the strategy developed in this study was called spike culture derived variant population (SCDV). The study provided proof-of-concept for such a strategy and we have used this approach to mutagenesis to develop durum wheat and barley variant populations (data not shown).

Correlation analysis of several morphological traits among the different SCDV lines revealed several interesting observations (Table 1, Figs S2-S4). The flag leaf length and the flag leaf angle were not correlated. However, the flag leaf sheath length and flag leaf length was significantly correlated $(p<0.001)$. Similarly, it was surprising that the flag leaf angle was not correlated with the seed weight per spike (Fig. S2), since it is known that erect flag leaf contributes to increased grain filling (Simon 1999). Nonetheless, it has to be pointed out that fewer variants with acute leaf angles were observed and among those many negatively affected seed weight or number due to reduced tillers, as reflected by few main tiller-bearing spikes (Fig. S3). There is one variant line of interest, 2C-3, which had acute flag leaf angle, but comparable to wild-type flag leaf length and flag leaf 
Table 1. Pearson correlation among traits measured in some variants of the SCDV population

\begin{tabular}{|c|c|c|c|c|c|c|c|}
\hline & $\begin{array}{c}\text { Flag leaf } \\
\text { length }\end{array}$ & $\begin{array}{l}\text { Flag leaf } \\
\text { angle }\end{array}$ & $\begin{array}{c}\text { Flag leaf } \\
\text { sheath } \\
\text { length }\end{array}$ & $\begin{array}{l}\text { Spike } \\
\text { length }\end{array}$ & $\begin{array}{l}\text { Length 1st } \\
\text { internode }\end{array}$ & $\begin{array}{l}\text { Length 2nd } \\
\text { internode }\end{array}$ & $\begin{array}{l}\text { Number of } \\
\text { seeds per } \\
\text { spike }\end{array}$ \\
\hline Flag leaf angle & -0.114 & & & & & & \\
\hline$p$-value & 0.138 & & & & & & \\
\hline $\begin{array}{l}\text { Flag leaf sheath } \\
\text { length }\end{array}$ & 0.494 & -0.056 & & & & & \\
\hline$p$-value & 0.000 & 0.465 & & & & & \\
\hline Spike length & 0.085 & 0.088 & 0.199 & & & & \\
\hline$p$-value & 0.267 & 0.251 & 0.008 & & & & \\
\hline Length 1 st internode & 0.415 & -0.048 & 0.449 & 0.094 & & & \\
\hline$p$-value & 0.000 & 0.531 & 0.000 & 0.214 & & & \\
\hline Length 2nd internode & 0.321 & -0.107 & 0.427 & -0.003 & 0.315 & & \\
\hline$p$-value & 0.000 & 0.162 & 0.000 & 0.968 & 0.000 & & \\
\hline $\begin{array}{l}\text { Number of seeds per } \\
\text { spike }\end{array}$ & 0.377 & -0.027 & 0.343 & 0.343 & 0.46 & 0.274 & \\
\hline$p$-value & 0.000 & 0.725 & 0.000 & 0.000 & 0.000 & 0.000 & \\
\hline $\begin{array}{l}\text { Weight of seeds per } \\
\text { spike }\end{array}$ & 0.319 & 0.034 & 0.296 & 0.326 & 0.444 & 0.287 & 0.862 \\
\hline$p$-value & 0.000 & 0.659 & 0.000 & 0.000 & 0.000 & 0.000 & 0.000 \\
\hline
\end{tabular}

sheath length, and had higher seed weight and seed number per spike than the wild-type. Furthermore, line 2C-3 had 10 spikes compared to 15 for the wild-type. Both internode lengths (Fig. S3), flag leaf length and flag leaf sheath length were significantly correlated $(p<0.001)$. The number of seed per spike and weight of seeds per spike were also significantly $(p<0.001)$ correlated to flag leaf length, flag leaf sheath length, spike length, and lengths of internodes. Of interest was the higher degree of correlation between the number of seeds per spike and the seed weight per spike with the first internode length than with the second internode. These findings concur with those reported for EMS-mutagenized wheat seeds (Dhaliwal et al. 2015). The data from sub-samples of the SCDV indicate a wide range of variability of morphological traits. The SCDV can be used to further characterize some of these variants and mining genes conferring the traits of interest.

\section{Molecular characterization of SCDV}

Methods for variant analysis include denaturing HPLC (McCallum et al. 2000a; b) and CelI digestion of heteroduplexes (Colbert et al. 2001). While the latter approach increased the throughput for screening including pooling DNA samples from individual plants 
(Colbert et al. 2001), the CelI-based screening was expensive it is less effective to identify mutations in genes with many small exons separated by large introns (Parry et al. 2009). More recently, alternative approaches using HRM (Ririe et al. 1997) and gel-based systems (Dong et al. 2009) have been demonstrated to be effective and less expensive. The presence of one mutation per $20.8 \mathrm{Kbp}$ in SbeIIb is higher than reported for the waxy genes mutation in wheat seed mutagenesis, where one mutation per $24 \mathrm{Kbp}$ was reported (Slade et al. 2005). Using a similar HRM screening approach on seed-derived EMS populations of wheat, based on SNP sequencing a mutation frequency of one per $40 \mathrm{Kbp}$ was detected in wheat Sbella (Botticella et al. 2011).

In conclusion, the strategy produced limited number of wasteful mutants and the manageable size of the SCDV lines enabled expeditious screening for mutations in genes of interest. In combination with HRM screening and sequencing strategies a large number of variants could be assessed as early as the $\mathrm{M}_{2}$ generation. With the recent demonstration of the mutation scanning approach in wheat using exon capture and next-generation sequencing (King et al. 2015), the detection of mutant events in the spike culture-derived variant population will be further expedited.

\section{Acknowledgements}

Canada Research Chairs and the Saskatchewan Agriculture Development Fund is acknowledged for financial support.

\section{References}

Botticella, E., Sestili, F., Hernandez-Lopez, A., Phillips, A., Lafiandra, D. 2011. High resolution melting analysis for the detection of EMS induced mutations in wheat SbeIIa genes. BMC Plant Biol. 11:156.

Campbell, J., Zhang, H., Giroux, M.J., Feiz, L., Jin, Y., Wang, M., Chen, X., Huang, L. 2012. A mutagenesisderived broad-spectrum disease resistance locus in wheat. Theor. Appl. Genet. 125:391-404.

Chen, L., Huang, L., Min, D., Phillips, A., Wang, S., Madgwick, P.J., Parry, M.A.J., Hu, Y.G. 2012. Development and characterization of a new TILLING population of common bread wheat (Triticum aestivum L.). Plos One 7:e41570.

Chibbar, R.N., Ganeshan, S., Baga, M. 2007. In planta novel starch synthesis. In: Ranalli, P. (ed.), Improvement of Crop Plants for Industrial Uses. Springer Verlag. Berlin, Germany. pp. 181-208.

Colbert, T., Till, B.J., Tompa, R., Reynolds, S., Steine, M.N., Yeung, A.T., McCallum, C.M., Comai, L., Henikoff, S. 2001. High-throughput screening for induced point mutations. Plant Physiol. 126:480-484.

Dhaliwal, A.K., Mohan, A., Sidhu, G., Maqbool, R., Gill, K.S. 2015. An ethylmethane sulfonate mutant resource in pre-green revolution hexaploid wheat. Plos One 10:e0145227.

Dong, C., Dalton-Morgan, J., Vincent, K., Sharp, P. 2009. A modified TILLING method for wheat breeding. The Plant Genome 2:39-47.

Ganeshan, S., Baga, M., Limin, A.E., Ratnayaka, I., Chodaparambil, S.V., Fowler, D.B., Chibbar, R.N. 2007. Biotechnological approaches for improving the tolerance of wheat to abiotic stress. In: Singh, D.P., Tomar, V.S., Behl, R.K., Upadhyaya, S.D., Bhale, M.S., Khare, D. (eds), Int. Conf. on Sustainable Crop Production in Stress Environments: Management and Genetic Options. Agrobios International. Jodhpur, India. pp. 297-309.

Ganeshan, S., Drinkwater, J.M., Repellin, A., Chibbar, R.N. 2010. Selected carbohydrate metabolism genes show coincident expression peaks in grains of in vitro-cultured immature spikes of wheat (Triticum aestivum L.). J. Agric. Food Chem. 58:4193-4201. 
Ganeshan, S., Leis, M., Drinkwater, J.M., Madsen, L.T., Jain, J.C., Chibbar, R.N. 2011. In vitro-cultured wheat spikes provide a simplified alternative for studies of cadmium uptake in developing grains. J. Sci. Food Agric. 92:1740-1747.

Henry, I.M., Nagalakshmi, U., Lieberman, M.C., Ngo, K.J., Krasileva, K.V., Vasquez-Gross, H., Akhunova, A., Akhunov, E., Dubcovsky, J., Tai, T.H., Comai, L. 2014. Efficient genome-wide detection and cataloging of EMS-induced mutations using exome capture and next-generation sequencing. Plant Cell 26:1382- 1397.

Hogg, A.C., Gause, K., Hofer, P., Martin, J.M., Graybosch, R.A., Hansen, L.E., Giroux, M.J. 2013. Creation of a high-amylose durum wheat through mutagenesis of starch synthase II (SSIIa). J. Cereal Sci. 57:377-383.

Jansson, C., Sun, C., Ganeshan, S., Chibbar, R.N. 2007. Plant breeding: antisense ODN inhibition in in vitro spike cultures as a powerful diagnostic tool in studies on cereal grain development. In: Esser, K., Lüttge, U.E., Beyschlag, W., Murata, J. (eds.), Progress in Botany. Springer Verlag. Berlin, Germany. pp. 179-190.

King, R., Bird, N., Ramirez-Gonzalez, R., Coghill, J.A., Patil, A., Hassani-Pak, K., Uauy, C., Phillips, A.L. 2015. Mutation scanning in wheat by exon capture and next-generation sequencing. Plos One 10:e0137549.

McCallum, C.M., Comai, L., Greene, E.A., Henikoff, S. 2000a. Targeting induced local lesions in genomes (TILLING) for plant functional genomics. Plant Physiol. 123:439-442.

McCallum, C.M., Comai, L., Greene, E.A., Henikoff, S. 2000b. Targeted screening for induced mutations. Nat. Biotechnol. 18:455-457.

Parry, M.A.J., Madgwick, P.J., Bayon, C., Tearall, K., Hernandez-Lopez, A., Baudo, M., Rakszegi, M., Hamada, W., Al-Yassin, A., Ouabbou, H., Labhilili, M., Phillips, A.L. 2009. Mutation discovery for crop improvement. J. Exp. Bot. 60:2817-2825.

Regina, A., Bird, A., Topping, D., Bowden, S., Freeman, J., Barsby, T., Kosar-Hashemi, B., Li, Z.Y., Rahman, S., Morell, M. 2006. High-amylose wheat generated by RNA interference improves indices of large-bowel health in rats. Proc. Natl Acad. Sci. U. S. A. 103:3546-3551.

Ririe, K.M., Rasmussen, R.P., Wittwer, C.T. 1997. Product differentiation by analysis of DNA melting curves during the polymerase chain reaction. Anal. Biochem. 245:154-160.

Sadasivaiah, R.S., Perkovic, S.M., Pearson, D.C., Postman, B. 2000. Registration of AC Nanda wheat. Crop Sci. 40:579-580.

Sestili, F., Botticella, E., Bedo, Z., Phillips, A., Lafiandra, D. 2010 Production of novel allelic variation for genes involved in starch biosynthesis through mutagenesis. Mol. Breed. 25:145-154.

Simon, M.R. 1999. Inheritance of flag-leaf angle, flag-leaf area and flag-leaf area duration in four wheat crosses. Theor. Appl. Genet. 98:310-314.

Slade, A.J., Fuerstenberg, S.I., Loeffler, D., Steine, M.N., Facciotti, D. 2005. A reverse genetic, nontransgenic approach to wheat crop improvement by TILLING. Nat. Biotechnol. 23:75-81.

Till, B.J., Reynolds, S.H., Greene, E.A., Codomo, C.A., Enns, L.C., Johnson, J.E., Burtner, C., Odden, A.R., Young, K., Taylor, N.E., Henikoff, J.G., Comai, L., Henikoff, S. 2003. Large-scale discovery of induced point mutations with high-throughput TILLING. Genome Res. 13:524-530.

Uauy, C., Paraiso, F., Colasuonno, P., Tran, R.K., Tsai, H., Berardi, S., Comai, L., Dubcovsky, J. 2009. A modified TILLING approach to detect induced mutations in tetraploid and hexaploid wheat. BMC Plant Biol. 9:115.

\section{Electronic Supplementary Material (ESM)}

Electronic Supplementary Material (ESM) associated with this article can be found at the website of CRC at http://www.akademiai.com/content/120427/

Electronic Supplementary Table S1. SSR primers and SBEIIb primers used for HRM screening

Electronic Supplementary Figure S1. Flag leaf length, flag leaf angle and flag leaf sheath length of variant lines of $\mathrm{M}_{2}$ plants compared to wild-type (black bar) 
Electronic Supplementary Figure S2. Length of spike (ear), first internode (from bottom of spike) and second internode of variants compared to wild-type (black bar)

Electronic Supplementary Figure S3. Number of seeds per spike, weight of seeds per spike and number of spikes (equivalent to number of main tillers from variants after compared to wild-type (black bar)

Electronic Supplementary Figure S4. Aligned melt curves from SSR-HRM analysis and variants SBEIIb sequences. (a) SSR marker GWM473; (b) PCR primers designed to amplify exon 3 of SBEIIb gene; (c) and (d) single base pair mutations in two independent variant lines compared to wild-type for the SBEIIb exon 3 